\title{
Satisfação e insatisfação no parto normal sob o enfoque dos atributos da qualidade da assistência
}

\author{
Satisfaction and dissatisfaction with normal birth from the care quality attributes standpoint \\ Satisfacción e insatisfacción en el parto normal bajo el enfoque de los atributos de la calidad de la asistencia
}

\begin{abstract}
Diana da Silva Gonçalves ${ }^{\circledR}$; Maria Aparecida Vasconcelos Moural ${ }^{\circledR}$; Adriana Lenho de Figueiredo Pereira ${ }^{\prime \prime} \oplus^{\circledR}$; Ana Beatriz Azevedo Queiroz' $\odot$; Clenilda Aparecida dos Santos' $\odot$; Hugo Demésio Maia Torquato' @
\end{abstract}

'Universidade Federal do Rio de Janeiro, Rio de Janeiro, RJ, Brasil; "Universidade do Estado do Rio de Janeiro, Rio de Janeiro, RJ, Brasil

\begin{abstract}
RESUMO
Objetivo: analisar os atributos da assistência ao parto normal relacionados com a satisfação e insatisfação na perspectiva de puérperas. Método: estudo qualitativo, com 30 puérperas, em maternidade do município do Rio de Janeiro, Brasil, realizada de outubro de 2019 a janeiro de 2020. As entrevistas foram submetidas à análise de conteúdo temática e referencial da estrutura da qualidade. Pesquisa aprovada por Comitê de Ética em Pesquisa. Resultados: a satisfação na assistência ao parto foi relacionada à comunicação eficaz, respeito à privacidade e autonomia com suporte emocional, convergentes com a estrutura da qualidade. A insatisfação foi atribuída à postura profissional desrespeitosa, à deficiência na infraestrutura e aos recursos no serviço. Considerações finais: escuta qualificada, assistência respeitosa e centrada na mulher foram basilares na qualidade percebida pelas participantes. Entretanto, a insatisfação foi relacionada à persistência de fragilidades na rede de atenção ao parto normal.
\end{abstract}

Descritores: Enfermagem; Saúde da Mulher; Parto Normal; Qualidade da Assistência à Saúde.

\begin{abstract}
Objective: to examine the attributes of birth care associated with childbearing women's satisfaction and dissatisfaction. Method: this qualitative study was conducted from October 2019 to January 2020 with 30 puerperal women at a maternity unit in the municipality of Rio de Janeiro, Brazil. The interviews were subjected to thematic content analysis and the quality structure frame of reference. The study was approved by the research ethics committee. Results: satisfaction with birth care was related to effective communication, respect for privacy, and autonomy with emotional support, which are convergent with a quality framework. Dissatisfaction was attributed to disrespectful professional behaviour, deficient infrastructure, and deficient service resources. Final Considerations: qualified listening, respectful and woman-centred care were crucial to participants' perceptions of quality. Dissatisfaction, meanwhile, was related to persistent shortcomings in the normal birth care system. Descriptors: Nursing; Women's Health; Natural Childbirth; Quality of Health Care.
\end{abstract}

\section{RESUMEN}

Objetivo: analizar los atributos de asistencia al parto relacionados con la satisfacción y la insatisfacción en la perspectiva de puérperas. Método: estudio cualitativo junto a 30 puérperas en una maternidad de Rio de Janeiro, Brasil, realizado de octubre de 2019 a enero de 2020. Se analizaron las entrevistas por contenido temático y referencial de la estructura de calidad. El Comité de Ética en Investigación aprobó la investigación. Resultados: la satisfacción en la asistencia al parto estuvo relacionada con la comunicación eficaz, respecto a la privacidad y autonomía con apoyo emocional, convergentes con la estructura de la calidad. La insatisfacción se atribuyó a la postura profesional irrespetuosa, la deficiencia de infraestructura y a los recursos del servicio. Consideraciones finales: escucha cualificada, asistencia respetuosa y centrada en la mujer fueron fundamentales en la calidad que las participantes mencionaron. Sin embargo, la insatisfacción estuvo relacionada con la persistencia de fragilidades en la red de atención al parto normal.

Descriptores: Enfermería; Salud de la Mujer; Parto Normal; Calidad de la Atención de Salud.

\section{INTRODUÇÃO}

A qualidade da assistência obstétrica ainda é um desafio no Brasil, em face da constatação de práticas inadequadas representadas pela elevada taxa de cesariana, que correspondeu a 55,9\% dos nascimentos em 2018, contrariando a taxa de $15 \%$ recomendada pela Organização Mundial da Saúde (OMS) ${ }^{1}$. Da mesma forma, condutas rotineiras de episiotomia, manobra de Kristeller, privação de alimentos e líquidos, restrição ao leito, desrespeito e maus-tratos suscitam a percepção de assistência agressiva e insatisfatória entre as mulheres ${ }^{2-8}$.

A satisfação da mulher com a assistência ao parto está relacionada à adequação das condições de atendimento, como a presença do acompanhante e percepção de protagonismo. No entanto, as intervenções destinadas à qualificação da

Agradecimentos à bolsa do Programa de Excelência Acadêmica - PROEX, Coordenação para a o Aperfeiçoamento de Pessoal de Nível Superior (CAPES, Brasil). Processo número 88887.465371/2019-00

Autora correspondente: Diana da Silva Gonçalves. E-mail: enfdiana.goncalves@gmail.com

Editora Científica: Cristiane Helena Gallasch; Editora Associada: Juliana Amaral Prata 
assistência ao parto raramente incluem a perspectiva da parturiente sobre a experiência vivenciada. Para que toda parturiente e recém-nascido recebam assistência de alta qualidade, a OMS estabeleceu atributos da melhoria da qualidade no parto e nascimento 9 .

A satisfação expressa a percepção pessoal positiva da assistência recebida no parto, evidenciando práticas e posturas profissionais implementadas à clientela. As expectativas prévias acerca do parto, quanto ao acesso ao cuidado obstétrico e às características do sistema de saúde, constituem medidas multidimensionais da qualidade que mostram a perspectiva da parturiente e possibilitam subsídios para melhoria da qualidade ${ }^{10}$. A insatisfação revela a perspectiva individual e desfavorável sobre elementos ou ausência de atributos influentes em danos físicos ou emocionais à puérpera e/ou ao recém-nascido ${ }^{4,11}$.

O movimento da humanização do parto considera esse período da vida reprodutiva feminina um evento fisiológico, preconiza o resgate da autonomia da mulher e o direito dela à assistência respeitosa ${ }^{12}$. Entretanto, a qualidade na assistência ao parto permanece como temática crítica na agenda de saúde materna e neonatal, com relação ao atendimento dos direitos humanos da mulher, os resultados de saúde e custos dos serviços.

Apesar dos progressos obtidos na assistência ao parto normal prestada por profissionais de saúde qualificados e nas condições estruturais mínimas dos serviços de saúde nas últimas décadas, a assistência obstétrica, ainda, precisa avançar na qualidade para obtenção de resultados exitosos e satisfatórios para as mulheres ${ }^{1,4,13}$. Em consideração a esse desafio, propôs-se o presente estudo, que objetivou analisar os atributos da assistência ao parto normal relacionados com a satisfação e insatisfação na perspectiva de puérperas.

\section{REFERENCIAL TEÓRICO}

O referencial teórico pauta-se na estrutura de qualidade proposta pela $\mathrm{OMS}^{13}$, que define os atributos essenciais dos serviços de saúde quanto aos recursos disponíveis e à assistência ao parto e ao nascimento, centrada na mulher, recém-nascido e família, aplicáveis em todos os serviços de maternidade. A utilização destes atributos possibilita orientação estratégica para implementação de melhorias e avaliação da qualidade da assistência nas instituições de saúde $^{13}$. A qualidade da assistência ao parto e nascimento é o grau em que os serviços de saúde proporcionam assistência coerente ao conhecimento científico atualizado, valorizando as preferências e os anseios da parturiente.

A qualidade do cuidado abarca duas dimensões do processo interrelacionadas: a oferta e a experiência do cuidado (Figura 1). A oferta do cuidado compreende as práticas assistenciais baseadas em evidências científicas, sistemas de informações e sistema de saúde que permita o encaminhamento para os diferentes níveis de atenção. A segunda dimensão é a experiência do cuidado e que balizou a presente pesquisa, contemplando a comunicação eficaz à parturiente e à família sobre a assistência prestada; a valorização de expectativas; e o respeito aos direitos, além do suporte emocional oferecido pela equipe ${ }^{13}$.

O quadro conceitual exposto na Figura 1 fornece a estrutura da qualidade da assistência e os componentes do processo assistencial, organizados em oito domínios que direcionam a avaliação e os aprimoramentos ${ }^{13}$.

As experiências do cuidado requerem comunicação eficaz entre profissional e parturiente para favorecer experiência positiva no parto. A equipe de saúde deve ter habilidades de aconselhamento prontamente acessíveis, atitude positiva e usar linguagem simples e clara para facilitar o entendimento e reconhecimento das necessidades de comunicação e preferências das mulheres ${ }^{13}$. A interação e a relação de confiança são necessárias ao processo assistencial satisfatório no trabalho de parto, bem como ambiente propício, com condutas adequadas e de acordo com as expectativas da cliente ${ }^{2}$.

Além desse atributo, torna-se essencial o respeito e a preservação da dignidade que envolvem a manutenção da privacidade, confidencialidade e ausência de maus-tratos na prestação da assistência. Portanto, busca-se motivar as parturientes na tomada de decisões informadas e esclarecimentos sobre as condutas e possíveis resultados ${ }^{13}$. 0 apoio emocional é também um atributo essencial para centralidade do cuidado na parturiente, a partir das necessidades individuais e da possibilidade de favorecer o protagonismo desta no trabalho de parto, com estímulo à presença de acompanhante de escolha e oferta de cuidados adequados, de acordo com a estrutura organizacional de cada instituição ${ }^{13}$ 


\section{Estrutura de qualidade da assistência da OMS para a saúde materna e neonatal}
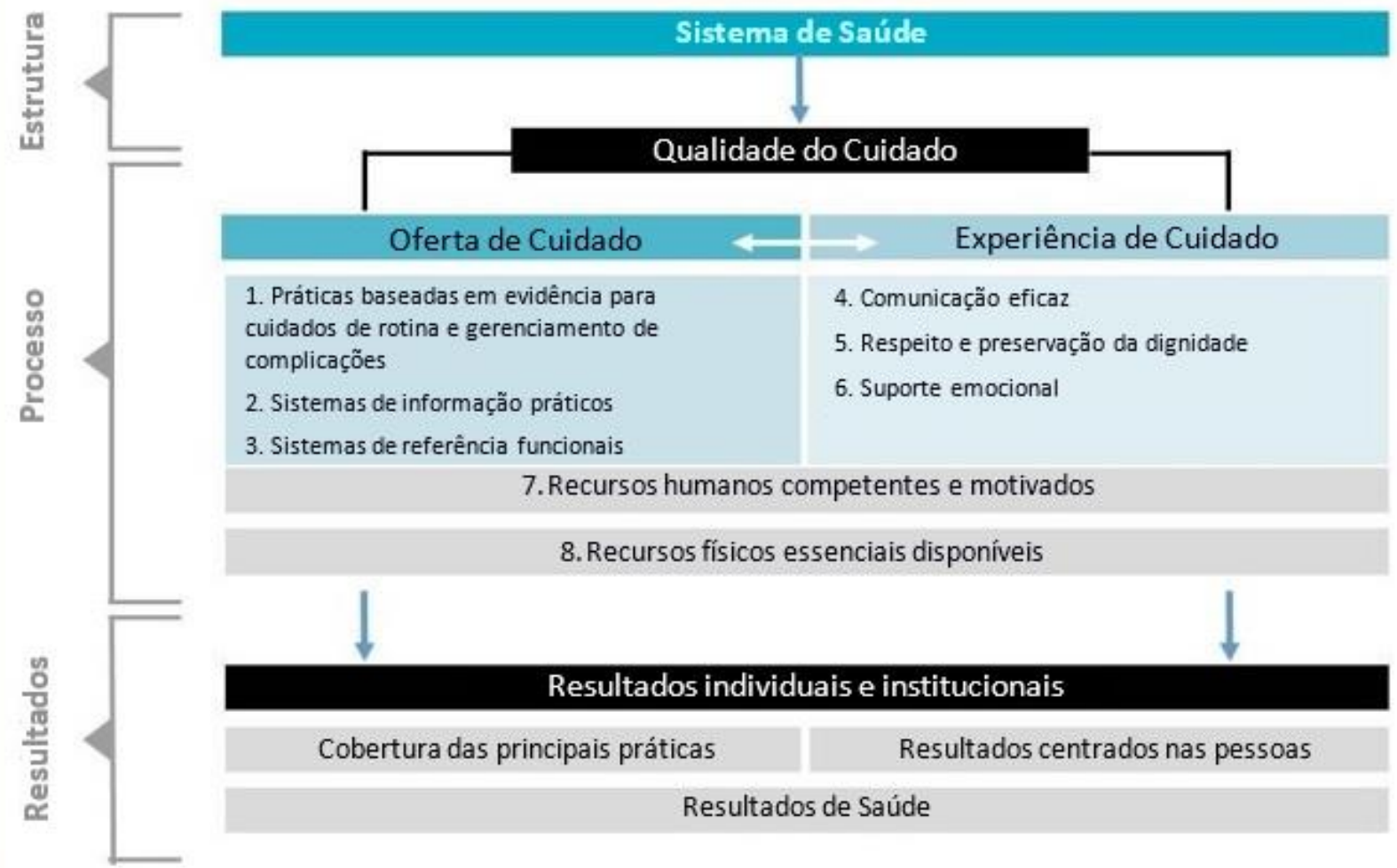

FIGURA 1: Estrutura da Qualidade da Assistência Materna e Neonatal proposta pela OMS. Rio de Janeiro, RJ, Brasil, 2021. Fonte: Adaptado de WHO. Standards for improving quality of maternal and newborn care in health facilities (2016).

\section{MÉTODO}

Estudo qualitativo, descritivo, realizado em maternidade municipal do Rio de Janeiro, Brasil. A maternidade foi selecionada por ser considerada referência na implementação das práticas de humanização do parto pela gestão municipal de saúde e contar com enfermeira obstétrica na assistência ao parto normal. Adotaram-se os critérios do Consolidated criteria for reporting qualitative research (COREQ) ${ }^{14}$.

As participantes do estudo foram 30 puérperas que deram à luz por parto normal, decorrente de gestação classificada de risco habitual. A seleção das participantes ocorreu mediante a identificação de características obstétricas no prontuário, identificando-se 48 puérperas elegíveis e que foram convidadas individualmente no Alojamento Conjunto $(\mathrm{AC})$, procedendo-se ao esclarecimento dos objetivos da pesquisa. Desta totalidade, 30 puérperas aceitaram participar do estudo e 18 delas recusaram.

Os critérios de inclusão foram puérperas com classificação de risco obstétrico habitual, que tiveram assistência ao parto normal prestada por enfermeiras e médicos obstetras e com pelo menos 24 horas de pós-parto. Excluíram-se aquelas com limitações na comunicação oral e desfecho de parto com óbito fetal ou neonatal precoce.

As entrevistas foram conduzidas pela pesquisadora principal, em sala reservada no AC, de outubro de 2019 a janeiro de 2020. Estas foram gravadas com o auxílio de gravador MP3, com duração média de 45 minutos, sendo transcritas na íntegra pela própria pesquisadora.

Utilizou-se de roteiro semiestruturado, previamente testado, sendo as entrevistas-piloto descartadas. Esse roteiro apresentava perguntas objetivas e subjetivas acerca do perfil sociodemográfico, histórico obstétrico e da assistência ao parto, concernente aos atributos da assistência envolvidos na satisfação e insatisfação com o atendimento recebido.

Utilizou-se da técnica de análise de conteúdo temática ${ }^{14}$, no processo de análise dos dados. Essa técnica visa descrever e interpretar o conteúdo das comunicações em geral, como o conjunto dos textos oriundos das entrevistas, possibilitando a compreensão dos elementos de significação, manifestos e acerca de um fenômeno social de interesse. 
A análise foi realizada em três fases sequenciais: pré-análise; exploração do material e tratamento dos resultados; inferência e interpretação ${ }^{14}$. As etapas de codificação e categorização ocorreram a partir de palavras ou expressões para alcance do núcleo de compreensão do texto, momento em que emergiram as categorias temáticas. Finalmente, o tratamento e a interpretação dos resultados permitiram o destaque das informações e análise à luz dos atributos da qualidade. As duas últimas fases da análise contaram com a supervisão de duas pesquisadoras não participantes da coleta de dados, a fim de dirimir inconsistências na análise.

O protocolo de pesquisa foi aprovado por Comitê de Ética em Pesquisa e obedeceu à legislação pertinente às pesquisas em seres humanos. $O$ anonimato foi preservado, por meio da identificação referente à entrevistada (E), seguida de numeral, conforme a ordem de realização, utilizando-se da denominação das participantes de E1 a E30.

\section{RESULTADOS E DISCUSSÃo}

Das 30 puérperas participantes do estudo, a maior parte delas estava com idade entre 20 e 34 anos ( $n=23$ ), convivia com o parceiro $(n=22)$ e se autodeclarou com cor da pele parda $(n=11)$ e preta $(n=8)$. Quanto ao grau de escolaridade, 13 puérperas concluíram o ensino médio e três possuíam o ensino superior completo. As demais puérperas estudaram até o ensino fundamental completo $(n=9)$ e incompleto $(n=4)$. A maioria $(n=18)$ das puérperas informou que a assistência ao parto normal foi conduzida por enfermeira obstétrica; as demais, por médico ( $n=3)$; e por ambos os profissionais $(n=4)$, sendo que quatro delas não souberam especificar a categoria do profissional.

A seguir, estão apresentadas e discutidas as duas categorias do estudo.

\section{Comunicação, protagonismo e acolhimento: a presença dos atributos da experiência do cuidado na satisfação da assistência ao parto normal}

Nessa categoria, evidencia-se que, sob a ótica dos atributos da qualidade, os elementos que corroboraram a satisfação da assistência recebida no parto normal se referiram a posturas e atitudes profissionais baseadas na comunicação efetiva. Este foi identificado como elo de vinculação e interação entre os profissionais, parturiente e acompanhante, resultando na sensação de conforto, segurança, confiança e liberdade à parturiente para expressão de necessidades e preferências ${ }^{13}$.

Os profissionais foram atenciosos, cautelosos e instruíram bem meu companheiro. Deixaram-me confortável e calma. A enfermeira foi excepcional (...) pedia licença quando realizava o toque [vaginal]. (E14)

Me senti à vontade para perguntar tudo e falei o que queria. As enfermeiras sempre incentivando, sem impor e se colocam no lugar do outro, criando vínculo. Foi maravilhoso. (E25)

A escuta qualificada como ferramenta de comunicação promoveu a sensação de zelo e disponibilidade profissional ao atendimento às necessidades da parturiente. Essa tecnologia relacional contribuiu para assistência de qualidade, uma vez que está ancorada em conhecimentos e habilidades técnicas científicas, em concordância com as recomendações ministeriais ${ }^{12}$. Utilizadas pelas enfermeiras obstétricas, possibilitaram a troca de informações e incentivo à participação da parturiente nas decisões da assistência.

A comunicação dos profissionais forneceu subsídios para que a parturiente participasse da assistência, sendo necessidades, desejos e escolhas observados e adotados como norteadores. A centralidade na parturiente contribuiu para o protagonismo, por se sentirem responsáveis pela condução do parto, vivenciando o empoderamento e a confiança, o qual excedeu à necessidade de intervenção médica. Nesta direção, a assistência centrada na parturiente foi referenciada como característica do atributo respeito e preservação da dignidade que proporcionou a sensação de tranquilidade e acolhimento.

Toda mulher e recém-nascido têm o direito de receber assistência respeitosa e digna, durante o período de internação hospitalar, o que inclui o respeito à privacidade, a confidencialidade das informações e a ausência de maustratos. Ademais, cita-se a dinâmica assistencial permeada pela tomada de decisão pela parturiente, a partir de informações claras fornecidas pelos profissionais de saúde ${ }^{1}$.

As falas mostraram, também, a autoconfiança quanto à naturalidade e potência para o parto, compreendida como experiência corporal, emocional e única.

Minha satisfação foi ser o centro daquele momento e o médico não ter imposto à vontade dele (...). Me senti segura, acolhida e respeitada, a mulher é quem faz o parto e os profissionais auxiliam no que for necessário. (E15)

O banho, ficar em pé e usar a bola facilitou (...) fui protagonista do meu parto. Trouxeram uma banqueta, coloquei a mão na vagina, senti a cabeça do bebê e fiz força. Foi um momento incrível. (E6)

O vínculo estabelecido no encontro com os profissionais expressou a percepção de acolhimento, privacidade e atenção. Experiência enriquecida pela liberdade de movimentos, posição, acesso ao chuveiro, banqueta de parto e bola 
bobath, tecnologias duras da assistência em saúde ${ }^{15}$, utilizadas a partir dos conhecimentos e das habilidades profissionais, os quais valorizaram a singularidade de cada parturiente.

Destaca-se a disponibilidade profissional em uma dinâmica de valorização da subjetividade, voltada para o atendimento das necessidades e dos anseios da parturiente, a qual ultrapassa as práticas rotineiras e normativas institucionais ${ }^{16}$.

Esses elementos, coadunados com as tecnologias leves em saúde, ou seja, aquelas concretizadas nas relações humanas, no modo de implementar e gerenciar o cuidado e os serviços ${ }^{15}$, emergiram como características do atributo respeito e preservação da dignidade, o que possibilitou o atendimento das necessidades e preferências no parto. Práticas favoráveis à autonomia da parturiente, ao propor posições confortáveis, seguras e alívio da dor, ao encontro das percepções de parturientes de São Paulo ${ }^{17,18}$, Minas Gerais ${ }^{19}$, Rio de Janeiro ${ }^{20}$, assim como no contexto internacional, como na Itália ${ }^{21}$, Austrália ${ }^{22}$ e Holanda ${ }^{23}$.

Verifica-se, portanto, que essas tecnologias de baixo custo, quando implementadas por profissionais competentes, são compatíveis com as percepções subjetivas da maioria das participantes, o que implicou satisfação e vivência positiva do parto normal hospitalar.

A assistência ao parto qualificada requer o reconhecimento do processo parturitivo como evento fisiológico e natural, ancorado no vínculo-terapêutico estabelecido entre profissional-parturiente-acompanhante. Nesta perspectiva, identificam-se as necessidades individuais para promoção de assistência singular que favoreça a satisfação da parturiente ${ }^{24,25}$

Ajudaram-me falando: 'faz a força' e deram orientação para o meu marido participar; e durante o parto, pude beber água e comer. Foi muito bom. (E23)

(...) Pari na banqueta, os profissionais tiveram paciência, estavam à minha volta e meu marido atrás; me senti protegida, respeitada e segura. Houve qualidade no atendimento, profissionalismo e cuidado. (E29)

O suporte emocional sensível às necessidades da parturiente propiciou a sensação de autoconfiança e segurança na competência dos profissionais que a assistiram. Esse atributo motivou e encorajou a livre movimentação, a adoção de posições confortáveis, a ingestão de alimentos e líquidos, com promoção do alívio da dor, conforto e favoreceu a autonomia da parturiente ${ }^{2}$. Abordagem que contribui para melhores experiências do parto, adesão aos cuidados pósparto, menor custo ao sistema de saúde, redução da mortalidade materna e promoção da melhoria da qualidade da assistência 26,27

A satisfação foi comumente associada à comunicação, à interação e ao envolvimento da parturiente e acompanhante na assistência, mediante informações claras e promoção de ambiente com redução de estímulo sonoros e luminosos. A decisão compartilhada ${ }^{12}$, o atendimento às expectativas e as preferências associados ao respeito convergiram aos resultados de uma revisão sistemática ${ }^{27}$.

O atual contexto obstétrico no Brasil caracteriza-se por um modelo híbrido, em que coexistem o paradigma humanizado e tecnocrático ${ }^{28}$. Nesta direção, evidenciou-se, por meio de relatos contrastantes, a insatisfação da assistência recebida frente à ausência dos atributos da qualidade.

\section{Vivência da insatisfação na não adequação aos atributos da qualidade reconfigurada na violência obstétrica}

Essa categoria evidenciou a ausência dos atributos da qualidade como fator interveniente na insatisfação da assistência recebida no parto. Esta percepção foi descrita a partir da postura profissional desrespeitosa, fragilidades na comunicação, recursos materiais e infraestrutura institucional precários.

A comunicação ineficaz entre os profissionais de saúde, parturiente e acompanhante resultou em preocupação e sensação de desassistência.

Tem umas profissionais que examinam, observam e vão embora, sem dizer nada, outras só ficam olhando, às vezes, de cara feia e meio ignorantes. (E7)

A assistência foi muito ruim. Já cheguei com dor para ganhar o bebê, mas não tinha ninguém para me acolher (...). Me senti como se fosse um lixo porque ninguém está ligando para nada. (E17)

A obstetra perguntou de onde eu vim, falei: de uma maternidade privada. Ela disse: Por que você veio de uma maternidade ótima para cá? (...) Os obstetras foram chatos, ali me julgando. Isso foi ruim, me travou. Era mais um atendimento para eles, mas para mim, um momento único. (E19)

As falas evidenciaram que essas participantes se sentiram vulneráveis diante da falha na comunicação, ausência de acolhimento e desvalorização de demandas. A exposição de preocupações e esclarecimentos foi inviabilizada, o que limitou a tomada de decisão informada e o exercício da autonomia. Os questionamentos e julgamentos sobre a escolha do local de parto foram identificados como desestimulantes e desrespeitos. Situação que caracteriza, segundo a OMS, assistência desrespeitosa, abusiva e negligente, desfavorável à experiência do parto ${ }^{13}$. 
A hipermedicalização do corpo feminino no evento parturitivo, descrita por essas parturientes, expressou características da violência obstétrica, reflexo da violência de gênero, fenômeno que demarca a violação dos direitos humanos das mulheres, realidade inserida na estrutura cultural e social de poder, a qual se apropria dos processos naturais femininos e a coloca em posição de passividade refletida nos serviços de saúde ${ }^{29}$.

Embora danoso, esse padrão de assistência ainda é recorrente, como relatado por parturientes de maternidades do Brasil $^{30}$, e outros países ${ }^{31,32}$ em pesquisas, o qual interfere na busca da assistência ao parto e nas baixas expectativas em partos subsequentes ${ }^{33}$

As limitações da infraestrutura do serviço e da rede de atenção obstétrica foram apontadas, também, como influentes no sentimento de insegurança, medo, mal-estar, frustração e desconforto.

Fiquei o tempo todo no chuveiro e a água foi para o quarto, o que deu trabalho, por isso, não gostei. Acho que o ambiente para a saúde da mulher deveria ter mais prioridade. (E6)

Foram três horas de dor, dúvida e insegurança (...), e me segurava nas barras e tentava sentar no chão, mas estava sujo. As condições do banheiro estavam ruins, devido à greve, toda expectativa desmoronou. (E19)

A carência de apoio da equipe obstétrica suscitou a sensação de sofrimento, desconforto e desassistência, momento em que se sentiram desamparadas e fragilizadas frente à ausência do alívio da dor ou suporte para o livre posicionamento. As restrições impostas a esse sensível momento confrontaram as expectativas da parturiente para um parto em ambiente adequado e a assistência recebida em condições precárias.

Essas participantes relataram fragilidades da infraestrutura do serviço de saúde, que as submeteram a condições desfavoráveis, durante o trabalho de parto, frente à crise do SUS. A situação política e econômica manifestada nas três esferas federal, estadual e municipal marcam um desafio para os gestores na organização das instituições diante da contenção financeira e precarização do sistema de saúde. Esta situação confere obstáculo para operacionalização das políticas públicas de saúde da mulher e qualificação da assistência ao parto normal ${ }^{34}$.

(...) Colocaram o soro, falaram que a dor iria melhorar, mas isso não aconteceu (...). Pedi cesárea, mas falaram que não, porque eu tinha passagem. Na hora que estava nascendo minha mãe, chamou a equipe, vieram correndo e quase não pegavam minha filha. Experiência péssima, sofri muito. (E 11)

Acho que teria que levar menos toque[vaginal] porque é muito desconfortável. (E 20)

Eu pari deitada com as pernas abertas e apoiadas na cama me segurando nas barras. Foi desconfortável, sentia câimbra, mas não tive opção (...). Não fizeram nada para aliviar a dor, não se importaram muito. (E 27)

A insatisfação frente à restrição de movimentos, toque vaginal frequentes e ausência da equipe profissional no período expulsivo evidenciam a assistência intervencionista e tecnocrática ainda recorrente no país ${ }^{21,35}$. Padrão assistencial que gerou insatisfação diante da limitação e exclusão de possíveis decisões, assim como o relato de mulheres de maternidades nas diferentes regiões do Brasil ${ }^{36}$, evidenciando paradigma danoso e insatisfatório, sob a perspectiva das mulheres. Nessa direção, a rejeição de intervenções desnecessárias e a busca pela assistência ao parto respeitosa, especialmente em ambiente fora dos hospitais, têm sido fenômeno de aumento gradual requerido pelas mulheres ${ }^{37}$.

Ademais, essas condutas aumentam o risco de dor no parto, desestimula a vivência do parto normal, favorece os distúrbios do sono, a alteração do humor e de apetite, o planejamento familiar para menos filhos, vínculo prejudicado com o recém-nascido, a decisão de não falar sobre a vivência do parto, o medo do parto normal e a identificação da cesárea como alternativa para sensação de segurança ${ }^{11}$. Essas consequências caracterizam-se por situações de violência institucional.

As participantes mencionaram atitudes profissionais negligentes, imprudentes e desrespeitosas, bem como a utilização inadequada de procedimentos e tecnologias assistenciais invasivas ao cuidado à saúde materna nos espaços hospitalares. Estas práticas desvalorizam a mulher como pessoa, tornando-a mero objeto da assistência e sem consideração para o sentimento de dor advindo dessas práticas, das condições físicas e estruturais inadequadas no serviço.

\section{Limitações do estudo}

As limitações do estudo ocorreram pelo desenvolvimento do estudo em única maternidade, pela coleta de informações no cenário da assistência e por contemplar um grupo particular de puérperas, o que impossibilita a generalização dos resultados aqui apresentados para outros cenários de assistência, em âmbito municipal e nacional.

\section{CONSIDERAÇÕES FINAIS}

Os elementos que conformaram a satisfação da assistência recebida no parto normal convergiram com os atributos da qualidade relacionados à experiência do cuidado, como a comunicação eficaz, o respeito e preservação da 
dignidade e o suporte emocional, descritos pela escuta qualificada e promoção do protagonismo da parturiente, em ambiente confortável com a integração do acompanhante durante o parto.

Embora os avanços na assistência obstétrica, progressivamente, promovam melhorias e alcance da satisfação na assistência recebida, evidencia-se, ainda, a necessidade de qualificação da assistência ao parto, pois a comunicação ineficiente, o desrespeito, a ausência de suporte emocional, as fragilidades de recursos materiais e humanos provocaram preocupação, sensação de sofrimento, desprezo, frustração e insatisfação. A violação dos direitos e da dignidade da parturiente evidenciaram a complexa relação entre as características do sistema de saúde, as práticas e posturas profissionais sobre a experiência no parto.

Apresentaram-se as potencialidades e os desafios na qualificação da assistência ao parto normal hospitalar no SUS, frente à inconsistência dos atributos da qualidade e persistência de elementos que desqualificaram a assistência, sob a perspectiva de parturientes.

\section{REFERÊNCIAS}

1. Vidal AT, Barreto JOM, Rattner D. Barriers to implementing childbirth recommendations in Brazil: the women's perspective. Rev Panam Salud Publica [Internet]. 2020 [cited 2021 Jan 15]; 44:e164. DOI: https://doi.org/10.26633/RPSP.2020.164.

2. Carvalho IS, Brito RS. Forms of obstetric violence experienced by mothers who had normal birth. Enferm Glob [Internet]. 2017 [cited $2021 \mathrm{Apr}$ 05]; 16(47):89-97. DOI: https://doi.org/10.6018/eglobal.16.3.250481.

3. Alemán NM. Between the formal and the substantive: quality of childbirth care in Uruguay. Sex Salud Soc [Internet]. 2017 [cited 2020 Nov 27]; 27:97-117. DOI: https://doi.org/10.1590/1984-6487.sess.2017.27.06.a.

4. Pedraza DF. Assistance to prenatal care, labor, and post-labor in the city of Campina Grande, Paraíba. Cad Saúde Coletiva [Internet]. 2016 [cited 2020 Oct 20]; 24(4):460-7. DOI: https://doi.org/10.1590/1414-462x201600040092.

5. Sena LM, Tesser CD. Obstetric violence in Brazil and cyberactivism of mothers: report of two experiences. Interface [Internet]. 2017 [cited 2020 Oct 22]; 21(60):209-20. DOI: https://doi.org/10.1590/1807-57622015.0896.

6. Betron ML, McClair TL, Currie S, Banerjee J. Expanding the agenda for addressing mistreatment in maternity care: a mapping review and gender analysis. Reprod Health [Internet]. 2018 [cited 2021 Jan 20]; 15(1):143. DOI: https://doi.org/10.1186/s12978-018-0584-6.

7. Benyamini Y, Molcho ML, Dan U, Gozlan M, Preis H. Women's attitudes towards the medicalization of childbirth and their associations with planned and actual modes of birth. Women Birth [Internet]. 2017 [cited 2020 Dec 13]; 30(5):424-30. DOI: https://doi.org/10.1016/j.wombi.2017.03.007.

8. Silva CM, Pereira BP, São Bento PAS, Vargens OMC The social interaction of puerperal women towards invasive childbirth procedures. Rev. enferm. UERJ [Internet]. 2020 [cited 2020 Dec 23]; 28:e52496. DOI: http://dx.doi.org/10.12957/reuerj.2020.52496.

9. Lazzerini M, Valente EP, Covi B, Semenzato C, Ciuch M. Use of WHO standards to improve quality of maternal and newborn hospital care: a study collecting both mothers' and staff perspective in a tertiary care hospital in Italy. BMJ Open Quality [Internet]. 2019 [cited 2020 Nov 02]; 8:e000525. DOI: http://dx.doi.org/10.1136/bmjoq-2018-000525.

10. Adnan Fl, Noor NM, Junoh NAM. Associated factors of labor satisfaction and predictor of postnatal satisfaction in the northeast of Peninsular Malaysia. PLoS ONE [Internet]. 2020 [cited 2021 Jan 15]; 15(8):e0238310. DOI: https://doi.org/10.1371/journal.pone.0238310.

11. Wigert H, Nilsson C, Dencker A, Begley C, Jangsten E, Sparud-Lundin C, Mollberg M, Patel H. Women's experiences of fear of childbirth: a metasynthesis of qualitative studies. Int J Qual Stud Health Well-being [Internet]. 2020 [ Cited 2020 Dec 28]; 15(1):1704484. DOI: https://doi.org/10.1080/17482631.2019.1704484.

12. Silva GF, Moura MAV, Queiroz ABA, Pereira ALF, Carvalho ALO, Netto LA. Opportunities for nurse midwives to bring change to the hegemonic model of obstetrics. Rev. enferm. UERJ [Internet]. 2020 [cited 2020 Dec 12]; 28:e49421. DOI: http://dx.doi.org/10.12957/reuerj.2020.49421.

13. World Health Organization (WHO). Standards for improving quality of maternal and newborn care in health facilities. Geneva: WHO; 2016.

14. Minayo MCSO. O Desafio do conhecimento: Pesquisa Qualitativa em Saúde. 12. ed. São Paulo: Hucitec; 2014.

15. Prata JA, Ares LPM, Vargens OMC, Reis CSC, Pereira ALF, Progianti JM. Non-invasive care technologies: nurses' contributions to the demedicalization of health care in a high-risk maternity hospital. Esc Anna Nery [Internet]. 2019 [cited 2020 Jul 15]; 23(2):e20180259. DOI: https://doi.org/10.1590/2177-9465-ean-2018-0259.

16. Rabelo ARM, Duarte ED, França BD, Silva KL. The care by obstetric nurses: the encounter between self care bodies and other woman who is cared for. Esc Anna Nery [Internet]. 2020 [cited 2020 Jul 15]; 24(1):e20190131. DOI: https://doi.org/10.1590/2177-9465-EAN-2019-0131.

17. Melo PS, Barbieri M, Westphal F, Fustinoni SM, Henrique AJ, Amorim A, Gabrielloni FMC. Maternal and perinatal parameters after non-pharmacological interventions: a randomised, controlled clinical trial. Acta Paul Enferm [Internet]. 2020 [cited 2020 Jul 15]; 33:eAPE20190136. DOI: https://doi.org/10.37689/acta-ape/2020A00136. 
18. Gallo RBS, Santana LS, Marcolin AC, Duarte G, Quintana SM. Sequential application of non-pharmacological interventions reduces the severity of labour pain, delays use of pharmacological analgesia, and improves some obstetric outcomes: a randomised trial. J Physiother [Internet]. 2018 [cited 2020 Jul 15]; 64(1):33-40. DOI: https://doi.org/10.1016/j.jphys.2017.11.014.

19. Dias EG, Ferreira ARM, Martins AMC, Nunes MMJ, Alves JCS. Efficacy of non-pharmacological methods for pain relief in labor normal of parturition. Enferm Foco [Internet]. 2018 [cited 2020 Jul 15]; 9(2):35-9. DOI: https://doi.org/10.21675/2357707X.2018.v9.n2.1398.

20. Vargens OMC, Silva ACV, Progianti JM. The contribution of nurse midwives to consolidating humanized childbirth in maternity hospitals in Rio de Janeiro-Brazil. Esc Anna Nery [Internet]. 2017 [cited 2020 Jul 15]; 21(1):e20170015. Available from: https://www.scielo.br/j/ean/a/dfNt7rwTQn7p63DYNMTC99q/?lang=en.

21. Lewis L, Hauck YL, Ronchi F, Crichton C, Waller L. Gaining insight into how women conceptualize satisfaction: Western Australian women's perception of their maternity care experiences. BMC Pregnancy Childbirth [Internet]. 2016 [cited 2020 Jul 17]; 16:29. DOI: https://dx.doi.org/10.1186\%2Fs12884-015-0759-x.

22. Steeghs VP, Lips SR, Warnaar TJS, Broerse JEW. Client-centred maternity care from women's perspectives: Need for responsiveness. Midwifery [Internet]. 2019 [cited 2021 Feb 04]; 74:76-83 DOI: https://doi.org/10.1016/j.midw.2019.03.016.

23. Ferrer BC, Jordana MC, Meseguer CB, García CC, Roche EM. Comparative study analysing women's childbirth satisfaction and obstetric outcomes across two different models of maternity care. BMJ Open [Internet]. 2016 [cited 2021 Jan 20]; 6:e011362. DOI: https://doi:10.1136/bmjopen-2016-011362.

24. Côrtes CT, Oliveira SMJV, Santos RCS, Francisco AA, Riesco MLG, Shimoda GT. Implementation of evidence-based practices in normal delivery care. Rev Latino-Am Enfermagem [Internet]. 2018 [cited 2020 Sep 25]; 26:e2988. DOI: https://doi.org/10.1590/1518-8345.2177.2988.

25. Amaral RCS, Alves VH, Pereira AV, Rodrigues DP, Silva LA, Marchiori GRS. Obstetric nursing and its interface with the brazilian obstetric model. Rev Enferm Atual in Derme [Internet]. 2019 [cited 2020 May 07]; 87. DOI: https://doi.org/10.31011/reaid2019-v.87-n.25-art.224.

26. Afulani PA, Smith ND, Phillips B, Sudhinaraset SSM. Validation of the person-centered maternity care scale in India. Reproductive Health [Internet]. 2018 [cited 2021 Jan 18]; 15:147. DOI: https://doi.org/10.1186/s12978-018-0591-7.

27. Macpherson I, Sánchez MVR, Legget FO, Fuertes F, Segarra I. A systematic review of the relationship factor between women and health professionals within the multivariant analysis of maternal satisfaction. Midwifery [Internet]. 2016 [cited 2021 Feb 03]; 41:68-78. DOI: https://doi.org/10.1016/j.midw.2016.08.003.

28. Leal MC. Childbirth and birth in Brazil: an evolving scenario. Cad Saúde Pública [Internet]. 2018 [cited 2021 Feb 03]; 34(5):e00063818. DOI: https://doi.org/10.1590/0102-311x00063818.

29. Sadler M, Santos MJ, Berdún DR, Rojas GL, Skoko E, Gillen P, Clausen JA. Moving beyond disrespect and abuse: addressing the structural dimensions of obstetric violence. Reprod Health Matters [Internet]. 2016 [cited 2020 Dec 18]; 24(47):47-55. DOI: https://doi.org/10.1016/j.rhm.2016.04.002.

30. Belfort IKP, Kalckmann S, Batista LE. Assistência ao parto de mulheres negras em um hospital do interior do Maranhão, Brasil. Saúde Soc [Internet]. 2016 [cited 2020 Dec 18]; 25(3):631-40. DOI: https://doi.org/10.1590/S0104-129020162571.

31. Sando D, Ratcliffe H, McDonald K, Spiegelman D, Lyatuu G, Sando MM, Emil F, Wegner MN, Chalamilla G, Langer A. The prevalence of disrespect and abuse during facility-based childbirth in urban Tanzania. BMC Pregnancy and Childbirth [Internet]. 2016 [cited 2021 Feb 05]; 16:236. DOI: https://doi.org/10.1186/s12884-016-1019-4.

32. Sethi R, Gupta S, Oseni L, Mtimuni A, Rashidi T, Kachale F. The prevalence of disrespect and abuse during facility-based maternity care in Malawi: evidence from direct observations of labor and delivery. Reproductive Health [Internet]. 2017 [cited 2021 Feb 01]; 14:111. DOI: https://doi.org/10.1186/s12978-017-0370-x.

33. Bobo FT, Kasaye HK, Etana B, Woldie M, Feyissa TR. Disrespect and abuse during childbirth in Western Ethiopia: Should women continue to tolerate? PLoS ONE [Internet]. 2019 [cited 2020 Feb 03]; 14(6):e0217126. DOI: https://doi.org/10.1371/journal.pone.0217126.

34. O'dwyer G, Graever L, Britto FA, Menezes T, Konder MT. Financial crisis and healthcare: the case of the municipality of Rio de Janeiro, Brazil. Ciênc Saúde Coletiva [Internet]. 2019 [cited 2020 Mar 26]; 24(12):4555-68. DOI: https://doi.org/10.1590/1413812320182412.23212019

35. Leal MC, Bittencourt SA, Pereira APE, Ayres BVS, Silva LBRAA, Thomaz EBAF, Lamy ZC, Pereira MN, Torres JA, Gama SGN, Domingues RMSM, Vilela MEA. Progress in childbirth care in Brazil: preliminar results of two evaluation studies. Cad Saúde Pública [Internet]. 2019 [cited 2020 Jul 23]; 35(7):e00223018. DOI: https://doi.org/10.1590/0102-311×00223018.

36. Apolinário D, Rabelo M, Wolff LDG, Souza SRRK, Leal GCG. Práticas na atenção ao parto e nascimento sob a perspectiva das puérperas. Rev Rene [Internet]. 2016 [cited 2020 Jul 17]; 17(1):20-8. DOI: https://doi.org/10.15253/21756783.2016000100004.

37. Happel-Parkins A, Azim KA. At pains to consent: A narrative inquiry into women's attempts of natural childbirth. Women Birth [Internet]. 2016 [cited 2020 Jul 17]; 29(4):310-20. DOI: https://doi.org/10.1016/j.wombi.2015.11.004. 